\title{
A Complementarity Resolution of the Black Hole Information Paradox
}

\author{
Mohamed S. El Naschie \\ Department of Physics, Faculty of Science, University of Alexandria, Alexandria, Egypt
}

Email address:

Chaossf@aol.com

To cite this article:

Mohamed S. El Naschie. A Complementarity Resolution of the Black Hole Information Paradox. American Journal of Astronomy and Astrophysics. Vol. 3, No. 5, 2015, pp. 77-86. doi: 10.11648/j.ajaa.20150305.11

\begin{abstract}
The E-infinity theory particle-wave duality used to compute the density of ordinary and dark energy of the cosmos is extended to meet the black hole complementarity of Susskind and 'tHooft. A black hole is essentially a relativistic as well as a quantum object. Therefore the information paradox of black holes is a consequence of the clash between these two most fundamental theories of modern physics. It is logical to conclude that a resolution of the problem requires some form of a quantum gravity theory. The present work proposes such a resolution using set theory and pointless spacetime geometry coupled to the afore mentioned extension and the inbuilt self referential character of Cantorian fractal sets.
\end{abstract}

Keywords: Black Holes Complementarity, S. Hawking, G. 'tHooft, L. Susskind, Transfinite Set Theory, Dvoretzky’s Theorem, Dark Energy, Self referential, Nano Casimir Reactor

\section{Introduction}

Let us "begin at the beginning" (although this is not a fantasy) and start playfully with drawing a round ring the size of a two pound sterling coin on an inflated large party balloon. When we let the air escape from this balloon we see how the ring becomes just a fat point. Without much ado, this is basically the point of this present paper, as we will see clearly by the time the reader has gone through twenty five per cent of the paper. Physically empty space with no matter, virtual particles or radiation what so ever is still far from being nothing $[1,2]$. Empty spacetime in the above sense is a sophisticated sloika, i.e. Mille-feuill of empty sets [1-20]. A point in such space is by no means the classical point defined somewhat naively as the intersection of two lines [7]. In fact a proper spacetime, which satisfies the physical quantum reality, is a points-less spacetime with inbuilt self referential fracal geometry and topology akin to that of von Neumann's continuous geometry $[76,119,132]$ as well as A. Conne's noncommutative geometry $[76,132]$ apart from the arch typical pointless space of E-infinity Cantorian spacetime [11132]. In such Cantorian-fractal geometry what appears to be a point is in actuality an entire Cantor set when magnified by sharpening the resolution of observation [33-130]. Combining the preceding fundamental insights gained from applying transfinite set theory and fractals to spacetime geometry with a fundamental theorem due to Aryeh Dvoretzky about measure concentration [65-67], we can argue that only 4.5 percent of the information inside a black hole could be regarded as inaccessible while the rest of the 95.5 percent of the information remains on the surface of the black hole no matter how much it shrinks because in the end analysis, spacetime has no ordinary points and no matter how small such points are, they are not zero nor can they vanish into nothingness because empty spacetime is anything but nothing. In fact empty spacetime is a multi-fractal made of infinitely many empty sets with varying degrees of emptiness $[1,2,12]$. In this sense we can reconcile what appeared for a considerable time irreconcilable, namely the view point of $\mathrm{S}$. Hawking [7] with all that speaks for it and speaks against it, with the clearly opposing views of L. Susskind and G. 'tHooft [133-139] which is understandable, logical and correct, but never the less does not propose an alternative waterproof answer to replace that of S. Hawking's famous but by no means entirely correct one [7].

The paper is subdivided into three reasonably short parts. First we introduce the required background information. Second we sketch our basic analysis and finally we summarize our conclusions. To keep the present work reasonably short we included a larger than average number of important references and included important papers on fractals and their self referential aspects in physics [140,141] which plays an important role in the present work. Last but 
not least it should be acknowledged from the outset that the recent revival of black hole physics and the information paradox at the prominent conference in Stockholm [133-135] as well as the highly influential writing of L. Susskind and G. 'tHooft [135-139] are the prime movers and motivation for the present work.

\section{Background Mathematical and Physical Information}

\subsection{Physical, Mathematical and Cosmological Measure Concentration Phenomena}

There are at least three well known types of measure concentration phenomena [64-67][140] relevant to the present work. The first is what we could call physical measure concentration. We could give two examples for that. The first is the Faraday cage demonstrating that for a metal sphere the total electric charge is concentrated on the surface with zero change inside the sphere [7]. This fact is behind the fortunate situation that an aeroplane flying in a thunder and lightening storm can protect the passengers from being electrocuted as is the case with a car with closed doors and windows. The second example is the energy of the quantum particle-wave where $4.5 \%$ of the energy is inside the quantum wave concentrated in the quantum particle as measurable ordinary energy while the quantum wave surrounding the quantum particles possesses most of the energy density, namely $95.5 \%$ as dark energy [14-61]. The best example for mathematical measure concentration is the marvellous Dvoretzky's theorem which states that in sufficiently high dimensionality, $96 \%$ of the volume of a sphere is concentrated near to its surface while the 'bulk' contains only $4 \%$ of the volume [140]. Finally cosmological concentration phenomena may be found theoretically in the fact that all information of a black hole is proportional to the surface and not to the volume of the black hole as demonstrated by the Bekenstein formula $[7,138]$. As a second example we could cite the work of the present author showing that the $4.5 \%$ ordinary energy of the universe can be measure 'inside' the universe while the rest, the $95.5 \%$ dark energy exists mainly near to the boundary of the holographic boundary of the universe [7,11]. It is the fact that our universe could be viewed as a giant black hole that we may argue that black holes, if they exist, will have most of its information, i.e. about $96 \%$ of the information on the surface of the black hole while $4 \%$ of the information could remain locked inside the black hole to which an outside observer will have no access.

\subsection{The Transfinite Theory of Spacetime}

To make a long, in fact very long story short, we start our journey to the exact picture of our physico-mathematical spacetime from the bijection formula $[74,75]$

$$
d_{c}^{(n)}=(1 / \phi)^{n-1}
$$

corresponding to von Neumann-Conne's dimensional function [74,75] of Penrose foliated universe, i.e. Penrose tiling. This is necessarily a fractal universe resembling a compactified holographic boundary, i.e. compactified Klein modular curve for a transfinite E8E8 Lie exceptional symmetry groups constituting our bulk $[74,75]$. Here $d_{c}^{(n)}$ is the Hausdorff dimension corresponding to $\mathrm{n}$ MengerUrysohn dimension and $\phi=2 /(1+\sqrt{5})$ is the Hausdorff dimension of the zero set, i.e. $\mathrm{n}=0$. To see that we set $\mathrm{n}=0$ in $d_{c}^{(n)}$ and find $[74,75]$

$$
\begin{aligned}
& d_{c}^{(0)}=(1 / \phi)^{0-1} \\
& =(1 / \phi)^{-1} \\
& =\phi
\end{aligned}
$$

The corresponding von Neumann-Conne formula is [76]

$$
D=a+b \phi
$$

where $\mathrm{a}, \mathrm{b} \in \mathrm{Z}$ and $\phi=(\sqrt{5}-1) / 2$. Setting $\mathrm{a}=0$ and $\mathrm{b}=$ 1 one finds the same value, namely

$$
\begin{aligned}
& D=0+(1)(\phi) \\
& =\phi \\
& =d_{c}^{(0)}
\end{aligned}
$$

This is our zero set which models the pre-quantum particle [129]

$$
D(Q P) \equiv(0, \phi)
$$

for the pre-quantum wave, i.e. the surface or cobordism of the quantum particle we just need to insert $n=-1$ which is the dimension of the neighbourhood of a point. That way we find $[74,75]$.

$$
\begin{aligned}
& d_{c}^{(-1)}=(1 / \phi)^{-1-1} \\
& =(1 / \phi)^{-2} \\
& =\phi^{2}
\end{aligned}
$$

or equivalently by setting $\mathrm{a}=1$ and $\mathrm{b}=-1$ one finds [7476]

$$
\begin{aligned}
& D(Q W)=1-\phi \\
& =\phi^{2} \\
& =d_{c}^{(-1)}
\end{aligned}
$$

This is the empty set which models the quantum particle. We see that the zero set $d_{c}^{(0)}$ separates the sets $d_{c}^{(n)}$ from the empty sets $d_{c}^{(-n)}$ and we are thus justified to speak of the degree of emptiness of an empty set [74-76] as we move 
from $\mathrm{n}=1$ to $\mathrm{n}=-2$ until we reach the truly insubstantial nothingness for $n=-\infty$ which leads to $d_{c}^{(-\infty)}=(1 / \phi)^{-\infty}=\phi^{\infty}=$ zero

\subsection{The Menger-Urysohn Dimensionality and the Dimensions of the Empty Set d(Menger $)=-1$}

The discussion of the section on the transfinite theory of spacetime depended crucially upon the extension of the notion of topological dimensions into the negative regime. In this section we show how simple, intuitive and easily grasped this extension of the deductive topological dimension theory which goes back to the Russian Paul Urysohn and the Austrian Karl Menger [74-76]. Take a 3D cube. The dimension of the cube is 3 but the dimension of the six sides of the surface of the cube is $D=3-2=1$ which is a trivial result. Applying the same argument to the two dimensional surface, the borders are lines and therefore we have $D=2-1=1$ which is equally trivial. Continuing this for the line we find that the dimensions of the edges of the line must follow the same formula, namely $D=n-1$. For a line $\mathrm{n}=1$ we find edge points $D=1-1=0$, again a trivial result. Here triviality stops because continuing for the point $n$ $=0$ one finds a non-trivial result, namely $D=0-1=-1$. Earlier on we called this the empty neighbourhood of a point or the empty set $[1,2]$. Continuing for $D=-\infty$ one finds then the truly completely empty set with the Hausdorff dimension $D^{(-\infty)}=(\phi)^{\infty}=$ zero and the bidimension representation [12,] [74-76]

$$
D(-\infty) \equiv(-\infty, 0)
$$

\section{An Exact Picture of Quantum Spacetime}

We clearly live in a $3+1=4$ dimensional world. These are three special dimensions plus a temporal dimension preventing things from happening all at once in some folkloristic philosophy. Einstein's special and general relativity takes the time dimension far more seriously as a dimension which one can put on equal footing as the space dimension if not even more [7]. Inserting $n=4$ in our bijection formula one finds [1, 2] [74-76]

$$
\begin{aligned}
& d_{c}^{(4)}=(1 / \phi)^{4-1} \\
& =(1 / \phi)^{3} \\
& =4+\phi^{3}
\end{aligned}
$$

Consequently we have [12,]

$$
D(4) \equiv\left(4 ; 4+\phi^{3}\right) \text {. }
$$

This means the fractal Hausdorff dimension is larger than the corresponding Menger-Urysohn topological dimension by the amount $\phi^{3}$ which we will see later on that it is equal to the intrinsic so called latent topological Casimir pressure of empty spacetime $[1,2]$. Now having found $4+\phi^{3}$ is an important result but we would like to scrutinize this result and analyse it in a far deeper way.

Let us look back at our spacetime as being made of an infinite mixture of all possible Cantor sets. That means starting from the unit set, i.e. classical line $d_{c}^{(1)}=1$ and the zero set $d_{c}^{(0)}=\phi$ until we reach the totally empty set. The sum of all these sets is clearly $[1,2]$

$$
\begin{aligned}
& \sum_{1}^{-\infty} d_{c}^{(n)}=d_{c}^{(1)}+d_{c}^{(0)}+d_{c}^{(-1)} \ldots . . \\
& =1+\phi+\phi^{2}+\phi^{3}+\ldots . \\
& =\frac{1}{1-\phi} \\
& =\frac{1}{\phi^{2}} \\
& =2+\phi
\end{aligned}
$$

Now since $d_{c}^{(0)}=\phi$ represents for us a random MauldinWilliams triadic Cantor set [1,2] living in one dimension, then gaging the sum $2+\phi$ in terms of this Cantor set, we can say that the dimension of our mixture of Cantor sets is simply $[1,2][74-76]$

$$
\begin{aligned}
& D=(2+\phi) / \phi \\
& =(2+\phi)(1 / \phi) \\
& =(2+\phi)(1+\phi) \\
& =\left(1 / \phi^{2}\right)(1 / \phi) \\
& =(1 / \phi)^{3} \\
& =4+\phi^{3}
\end{aligned}
$$

which is the Hausdorff dimension of our spacetime. However there is a still more profound interpretation of these results because $1+\phi=1 / \phi$ is the inversion of the Hausdorff dimension $\phi^{2}$ of a quantum particle while $(1 / \phi)^{2}=2+\phi$ is the inversion of the Hausdorff dimension of the quantum wave $\phi^{2}$. In other words $4+\phi^{3}$ is both the intersection between the particle like behaviour given by $(1 / \phi)=1+\phi$ and the wave like behaviour dimension $(1 / \phi)^{2}=2+\phi$

$$
(1+\phi)(2+\phi)=4+\phi^{3}
$$

as well as the union of the two, namely

$$
(1+\phi)+(2+\phi)=4+\phi^{3}
$$


where $(1+\phi)$ and $(2+\phi)$ are the un-normed probability of particle and wave respectively. In other words our space is blind to the union and intersection and cannot tell them apart which is the deepest explanation possible for the particlewave duality of quantum mechanics $[1,2]$. Armed with all the preceding results let us see if we can construct a simple exact picture of micro spacetime.

We start with the zero set pre-particle. This is

$$
D(0)=(0, \phi)
$$

Surrounding $D(0)$ is its cobordism or the guiding quantum wave which means the empty set $[1,2]$

$$
D(-1)=\left(-1, \phi^{2}\right)
$$

The cobordism, or surface of the guiding Bohm-Einstein quantum wave, is clearly $D(-2)$ which is given by $[1,2]$

$$
D(-2)=\left(-2, \phi^{3}\right)
$$

Now something quite remarkable happens at this point. The particle wave entity is floating in spacetime with an average dimension $4+\phi^{3}$ which means a normed average dimension $1 /\left(4+\phi^{3}\right)=\phi^{3}$. That means spacetime is the cobordism, i.e. the surface of the guiding Bohm-Einstein, ergo ghost quantum wave and consequently the surface of the quantum wave is given by the expectation value [2]

$$
\langle D(-2)\rangle=\left\langle(-2), \phi^{3}\right\rangle .
$$

In other words our quantum spacetime is nothing but a zero set pre-quantum particle surrounded by an empty set pre-quantum wave floating in the surface of the quantum wave given by the expectation value $\left\langle-2, \phi^{3}\right\rangle$. The ghost is no ghost at all unless we consider spacetime to be a ghost. In the next section we will see how al of that relates to Casimir energy and dark energy [2].

\section{The Casimir Local Topological Pressure}

If two plates which are conducting but uncharged are put very close vis-à-vis each other, it is an experimental fact that they are pulled together by what is known as the Casimir effect $[94,120,121]$. The E-infinity set theoretical explanation advanced some time ago is as follows: The nano distance between the two plates is as near as we can come to create an empty set. That means inside the plates we have a topological pressure $\phi^{2}$ stemming from the state of the entropy-like disorder measured by the Hausdorff dimension $\phi^{2}$ of the empty set. Outside, adjacent to the plates, we have the zero set of the quantum particles. Consequently the net topological pressure is the difference between the empty set quantum wave $\phi^{2}$ and the zero set quantum particle which means $\phi-\phi^{2}=\phi^{3}$ giving us the intrinsic latent topological pressure of empty spacetime which by inversion, leads to the Hausdorff dimension $4+\phi^{3}$. In other words the Casimir topological pressure is the same at the counter factual part in the general formula for Hardy's quantum entanglement, namely [58][68-71]

$$
P=\phi^{n+3}
$$

and setting the number of particles $n=0$ we are left with the global part [1-34]

$$
P=\phi^{3} .
$$

Thus we could think of $\phi^{3}$ as the excess in the fluctuation of spacetime dimension $4+\phi^{3}$ over the average topological dimension 4 causing the extra pressure of $\phi^{3}$ which manifests itself locally as the Casimir effect. A pedestrian way to understand that is the following: The un-normalized probability due to quantum particles is $1 / \phi=1+\phi$ and for the quantum wave is $1 / \phi^{2}=2+\phi$. The minimal integer approximation of the sum of both is clearly $1+2=3$ and the maximal is $3+2=5$. Consequently the average integer value is $(3+5) / 2=4$. On the other hand $\mathrm{D}$ is not 4 but $4+\phi^{3}$ showing that $\phi^{3}$ is indeed a measure for topological dimensional or entropic fluctuation in full agreement with all previous conclusions which we made earlier on [7][11-20].

\section{Dark Energy is Global Dvoretzky Concentration of Casimir Energy}

The two Casimir plates of the classical Casimir effect experiment, plays obviously the role of a boundary condition. What happens then when this boundary is pushed to infinity? The answer is it becomes the boundary of the holographic boundary of the universe [7]. This boundary is obviously a one sided boundary with nothing outside to push back to create a statical balance. It is a Möbius-like multidimensional boundary and consequently the universe must expand into the insubstantial nothingness surrounding our universe which is a clopen (which means open and closed topologically) and a multiverse of its own self. Considering that this E-infinity multiverse universe has formally infinite dimensions, we see that the Dvoretzky theorem [140] will apply and as a result $95.5 \%$ of the energy of this universe will be concentrated at the edge of the universe. Consequently the difference between the Casimir energy and dark energy is the difference between local and global as well as two sided and one sided boundary conditions [120-127]. 


\section{How to Build a Nano Casimir-Dark Energy Reactor}

From the preceding discussion it is an inescapable conclusion that we could build a nano universe using nano technology and that such a universe will constitute a unit cell of a nano Casimir-dark energy reactor [94,96,120] from which we can extract an infinite amount of clean energy using empty spacetime as our fuel.

To start we can use a large amount of $\mathrm{C}_{60}$ Fullerene [7] nano particles which are the smallest dodecahedron [7] known to exist at present. There are many reasons to suspect that the universe geometry and topology resembles that of a dodecahedron so that each $\mathrm{C}_{60}$ particle is an approximately universe prototype geometrically and topologically speaking [7]. Each two faces of the $\mathrm{C}_{60}$ powder will act as a Casimir cell and the complexity of the heap will produce an artificial local high dimensionality for which Dvoretzky's theorem applies [140]. Adding a large number of these artificial nano universes we end up with a macroscopic multiverse from which energy can in principle be extracted from its most outer surface. The rest is technological details. However we are more than aware that the devil lies in the detail. Never the less all that we can say at this point is that we will use in principle the same technology used to minimize the usually harmful effects of the Casimir effect on nano devices only this time in a reversed fashion, i.e. trying to maximise these effects rather than minimizing it [120-127].

\section{A set Theoretical Resolution of the Black Hole Information Paradox}

We started by discussing black holes, then moved from there to Casimir energy, dark energy and nano reactors [140]. With the benefit of hindsight let us see what the connection is between these seemingly very different problems. Maybe it is good for a deep understanding of the problem at hand to ask oneself can we really solve a mind teaser like the information paradox without knowing really what space is, what time is and worse still, what the word nothingness means. The present author admits that he thought that this is not possible and that was exactly his point of departure. First of all totally empty space is not nothing but something substantial even without any fluctuation or pair creation and annihilation. Empty spacetime is a multidimensional empty set. The empty set is far from being nothing. As soon as you mention the word set then the word nothing is not there. Insubstantial nothingness is not even a set. The border line is the totally empty set given by $(\phi)$ to the power of (infinity). The empty set on the other hand is given by two dimensions, namely minus one and $\phi^{2}$ both of which are not zero or nothing. Consequently by shrinking a spherical black hole indefinitely it can never become a zero point with a zero surface area because quantum space has no ordinary (naïve) classical point exactly as the pointless spaces of von Neumann's noncommutative geometry and E-infinity Cantorian spacetime [7]. The information density becomes extremely large but not infinity as the black hole becomes extremely small but never zero so that at the end about $95.5 \%$ of the information encoded on the surface of the black hole is never lost and only $4.5 \%$ of the information inside the black hole becomes inaccessible to us. Paradoxically for us living inside this giant black hole we call the universe, the situation is reversed for something related to information, via entropy which we call energy [120-127]. Only $4.5 \%$ of the energy of the universe is accessible to us while $95.5 \%$ of the energy of the universe cannot be measured directly and we known that it is there only because of observing its effect manifested via the accelerated cosmic expansion [140].

\section{Discussion: The Ordinary Information-Dark Information Complementarity}

\subsection{General Discussion and Remarks}

The fact that a resolution of the black hole information calls for a quantum gravity theory was too obvious to escape the attention of all the expertise on the subject with L. Susskind and G. 'tHooft at the very front. For those working on fractal spacetime it was equally obvious from the beginning that a theory of quantum gravity requires a theory of spacetime which reconciles Einstein's spacetime of relativity with the traditional spacetimeless quantum mechanics of Bohr and Heisenberg [1-7]. Those coming from the school of fractal spacetime think the answer to the above is too simple to see at once. It is self similarity which has the key to quantum gravity and consequently the information paradox by illuminating both the zero and infinity from our computation while giving them a prominent welcome in our physico-philosophical model building conception $[74,75]$. This point will take us first into deep water involving complementarity, particle-wave duality and measure concentration theorems but we think after that we will reach a secure shore of basic understanding of the Einstein program of unification and geometrical visualization [68-71].

\subsection{Four Dimensionality and Self Similarity}

Four dimensionality is nature's way of keeping a family secret under sealed lips. The mild shame of harbouring infinite dimensions is no secret however for those working in Einfinity Cantorian-fractal spacetime [80-84]. There is no doubt that zero and infinity are non-numbers which were behind much of the trouble with high energy and fundamental quantum physics until there were tamed by the young Dutch physicist with an unusual name to spell, Gerardus 'tHooft, to match his unusual way of thinking mathematically first and physically second. We need to recall two obvious facts in this connection. First any two different numbers multiplied or divided always results in a different third number. The only exceptions are zero and infinity. Any number multiplied by 
zero or infinity is zero or infinity. Again the second exception is zero and infinity themselves where zero multiplied with infinity is undefined. To escape this trouble easily God gave us the most fundamental fractals with what became known as self similarity or more generally, self affinity and self referentiality [141]. With random Cantor sets we escape for ever from burdening our computers and our brains with zero and infinity. They come with an amazing transfinite Turing computer dubbed in the fractal Cantorian spacetime trade as the golden mean computer [21][83]. Using this new mathematicalgeometrical-topological facilities, we can renormalize the black hole singularity so to speak and get rid of the information paradox as a paradox in unheard of simplicity.

The second point is that self similarity implies that every point in our universe, upon sufficient magnification similar to the balloon example mentioned at the very beginning of the introduction of the present paper, is again our universe so that this universe can be seen as a black hole quasi singularity. This self referential complementarity is a major player in resolving the black hole information paradox. Consequently we are living inside a black hole universe which is necessarily a multiverse. That way we are gently led to validate the work of not only L. Susskind and G. 'tHooft but also a substantial part of S. Hawking's conjectured results [7][133-142]. Thus the self referential complementarity is also the answer to G. 'tHooft's question on Research Gate [142].

\subsection{Black Hole Complementarity and the Mystery of Dark Energy}

Those familiar with the black hole information paradox are surely familiar with L. Susskind and G. 'tHooft's complementarity resolution which reconciles S. Hawking's views with fundamental physics [136, 137]. Well, following this line of reasoning, our present solution is a detailed version of the said complementarity solution. We recall first that a black hole need not be that of Schwarzs child's one but we can deal with it much better as the exact solution obtained long ago by Kerr. Seeing it that way we can go one step further and think of the generalization of $\mathrm{E}=\mathrm{mc}^{2}$ within a quasi quantum gravity theory such as that of Mageuijo-Smolin theory or the equivalent E-infinity theory which sees $\mathrm{E}$ as a double Eigen value problem corresponding to a doubly special relativity theory with the speed of light $\mathrm{c}$ and the Planck length Lp playing a similar role simultaneously. That way the present author reached the conclusion that the $4.5 \%$ ordinary measurable energy and the $95.5 \%$ dark energy [38-44], which we cannot measure directly are complementary. This complementarity follows more or less directly from the waveparticle duality as well as geometrically from the incredible measure theoretical theorem of Dvoretzky [65] [140].

\subsection{Dark Energy and Dark Information}

Adding to the above discussion the elementary fact that information and energy are proportional via entropy, it is an equally trivial although slightly terse conclusion to realize that dark energy is deeply connected to the information paradox of black holes and that the lost information is not really lost but only not directly observable in the same way as dark energy, which is connected to the quantum wave zero set is not directly observable $[11,12]$. The similarity between this line of argument and the Susskind and 'tHooft black hole complementarity is obvious. In this sense we may coin a new word, namely dark information.

\section{Conclusion}

We started with a trivial expanding and shrinking balloon demonstrating that what seems to be a point is scale dependant observation and ended by reinforcing the black hole complementarity argument of L. Susskind and G. 'tHooft. Our conclusion is that actual empty spacetime is far from being nothing and is a multi-dimensional empty set, which is a substantial something. This spacetime is pointless and what appears to be a point is, upon magnification, a complete random Cantor set. In such spacetime a black hole will always have a non-zero surface area no matter how small it shrinks and will never vanish and have a zero surface area. In such a situation, and by a well known brilliant theorem due to the great late A. Dvoretzky who was at a time the President of the Wiseman Institute, at least $95.5 \%$ of the information of a black hole will not be lost. The situation is analogous to that of the ordinary and the dark energy of the universe and could be used as a guiding principle in the design of a nano Casimir-dark energy reactor. In the end analysis we think that our inability to give a waterproof definition to the words point, line and so on coupled with mildly ignoring the impact of nonlinear dynamics, chaos and the self referential character of fractals on fundamental quantum physics contributed to a delay of the development of black hole research and related subjects. Another source of misunderstanding is the wrong extrapolation from lower dimensionality to high dimensionality which leads to the erroneous conclusion that it will dilute the density of information at the holographic boundary surface while by Dvoretzky's theorem, it is the other way around [124]. We hope the present work has at a minimum helped positively in this direction by reinforcing the complementarity resolution of the black hole information paradox using entirely different mathematics and showing that the paradox is no longer a paradox.

\section{References}

[1] L. Marek-Crnjac: Cantorian Space-Time Theory: The Physics of Empty Sets in Connection With Quantum Entanglement and Dark Energy. Lambert Academic Publishing, Saarbrucken, Germany. ISBN: 978-3-659-12876-9, 2013.

[2] Mohamed S. El Naschie: Quantum fractals and the Casimirdark energy duality - The road to a clean quantum energy nano reactor. Journal of Modern Physics, 6, 2015, pp. 13211333.

[3] M. S. El Naschie: Some tentative proposals for the experimental verification of Cantorian micro spacetime. Chaos, Solitons \& Fractals, 9(12), 1998, pp. 143-144. 
[4] P. Kumar Chattaraj (Editor): Quantum Trajectories. CRC Press. Taylor \& Francis Group, Boca Raton, USA, 2011.

[5] M. S. El Naschie: The fractal dimension of spacetime - Remarks on theoretical derivation and experimental verification. Chaos, Solitons \& Fractals, 9(7), 1998, pp. 1211-1217.

[6] Sir Herman Bondi: Relativity and the Commonsense. A New Approach to Einstein. Heinemann, London, 1964.

[7] R. Penrose: The Road to Reality. A Complete Guide to the Laws of the Universe. Jonathan Cape, London, 2004.

[8] L. B. Okun: Energy and mass in relativity theory. World Scientific, Singapore 2009.

[9] W. Rindler: Relativity (Special, General and Cosmological). Oxford University Press, Oxford. 2004.

[10] Mohamed S. El Naschie: On a New Elementary Particle from the Disintegration of the Symplectic 'tHooft-Veltman-Wilson Fractal Spacetime. World Journal of Nuclear Science and Technology, 4(4), 2014, pp. 216-221.

[11] M. A. Helal, L. Marek-Crnjac, Ji-Huan He: The Three Page Guide to the Most Important Results of M. S. El Naschie's Research in E-Infinity Quantum Physics and Cosmology. Open Journal of Microphysics, 3(4), 2013, pp. 141-145.

[12] L. Marek-Crnjac, Ji-Huan He: An Invitation to El Naschie's Theory of Cantorian Space-Time and Dark Energy. International Journal of Astronomy and Astrophysics, 3(4), 2013, pp. 464-471.

[13] Jean-Paul-: E-Infinity Dualities, Discontinuous Spacetimes, Xonic Quantum Physics and the Decisive Experiment. Journal of Modern Physics, 5(15), 2014, pp. 1427-1436.

[14] Mohamed S. El Naschie: Quantum Entanglement as a Consequence of a Cantorian Micro Spacetime Geometry. Journal of Quantum Information Science, 1(2), 2011, pp. 50-53.

[15] Mohamed S. El Naschie: A Resolution of Cosmic Dark Energy via a Quantum Entanglement Relativity Theory. Journal of Quantum Information Science, 3(1), 2013, pp. 23-26.

[16] M. S. El Naschie: A Unified Newtonian-Relativistic Quantum Resolution of the Supposedly Missing Dark Energy of the Cosmos and the Constancy of the Speed of Light. International Journal of Modern Nonlinear Theory and Application, 2(1), 2013, pp. 43-54.

[17] Mohamed S. El Naschie: Quantum Entanglement: Where Dark Energy and Negative Gravity plus Accelerated Expansion of the Universe Comes From. Journal of Quantum Information Science, 3(2), 2013, pp. 57-77.

[18] Mohamed S. El Naschie: A Fractal Menger Sponge SpaceTime Proposal to Reconcile Measurements and Theoretical Predictions of Cosmic Dark Energy. International Journal of Modern Nonlinear Theory and Application, 2(2), 2013, pp. 107-121.

[19] Mohamed S. El Naschie: The Hydrogen Atom Fractal Spectra, the Missing Dark Energy of the Cosmos and Their Hardy Quantum Entanglement. International Journal of Modern Nonlinear Theory and Application, 2(3), 2013, pp. 167-169.

[20] Mohamed S. El Naschie: A Rindler-KAM Spacetime Geometry and Scaling the Planck Scale Solves Quantum Relativity and Explains Dark Energy. International Journal of Astronomy and Astrophysics, 3(4), 2013, pp. 483-493.
[21] M. S. El Naschie: What Is the Missing Dark Energy in a Nutshell and the Hawking-Hartle Quantum Wave Collapse. International Journal of Astronomy and Astrophysics, 3(3), 2013, pp. 205-211.

[22] Mohamed S. El Naschie: The Missing Dark Energy of the Cosmos from Light Cone Topological Velocity and Scaling of the Planck Scale. Open Journal of Microphysics, 3(3), 2013, pp. 64-70.

[23] Mohamed S. El Naschie: From Yang-Mills Photon in Curved Spacetime to Dark Energy Density. Journal of Quantum Information Science, 3(4), 2013, pp. 121-126.

[24] Mohamed S. El Naschie: Calculating the Exact Experimental Density of the Dark Energy in the Cosmos Assuming a Fractal Speed of Light. International Journal of Modern Nonlinear Theory and Application, 3(1), 2014, pp. 1-5.

[25] Mohamed S. El Naschie: Cosmic Dark Energy Density from Classical Mechanics and Seemingly Redundant Riemannian Finitely Many Tensor Components of Einstein's General Relativity. World Journal of Mechanics, 4(6), 2014, pp. 153-156.

[26] Mohamed S. El Naschie: Capillary Surface Energy Elucidation of the Cosmic Dark Energy - Ordinary Energy Duality. Open Journal of Fluid Dynamics, 4(1), 2014, pp. 15-17.

[27] Mohamed S. El Naschie: Einstein's General Relativity and Pure Gravity in a Cosserat and De Sitter-Witten Spacetime Setting as the Explanation of Dark Energy and Cosmic Accelerated Expansion. International Journal of Astronomy and Astrophysics, 4(2), 2014, pp. 332-339.

[28] Mohamed S. El Naschie: Electromagnetic-Pure Gravity Connection via Hardy's Quantum Entanglement. Journal of Electromagnetic Analysis and Applications, 6(9), 2014, pp. 233-237.

[29] Mohamed S. El Naschie: Cosmic Dark Energy from 't Hooft's Dimensional Regularization and Witten's Topological Quantum Field Pure Gravity. Journal of Quantum Information Science, 4(2), 2014, pp. 83-91.

[30] Mohamed S. El Naschie: Entanglement of E8E8 Exceptional Lie Symmetry Group Dark Energy, Einstein's Maximal Total Energy and the Hartle-Hawking No Boundary Proposal as the Explanation for Dark Energy. World Journal of Condensed Matter Physics, 4(2), 2014, pp. 74-77.

[31] Mohamed S. El Naschie: The Meta Energy of Dark Energy. Open Journal of Philosophy. 4(2), 2014, pp. 157-159.

[32] Mohamed S. El Naschie: Pinched Material Einstein Space-Time Produces Accelerated Cosmic Expansion. International Journal of Astronomy and Astrophysics. 4(1), 2014, pp. 80-90.

[33] Mohamed S. El Naschie: From Chern-Simon, Holography and Scale Relativity to Dark Energy. Journal of Applied Mathematics and Physics, 2(7), 2014, pp. 634-638.

[34] Mohamed S. El Naschie: Why E Is Not Equal to mc2. Journal of Modern Physics, 5(9), 2014, pp. 743-750.

[35] Mohamed S. El Naschie: Nash Embedding of Witten's M-Theory and the Hawking-Hartle Quantum Wave of Dark Energy. Journal of Modern Physics, 4(10), 2013, pp. 1417-1428.

[36] Mohamed S. El Naschie: Dark Energy from Kaluza-Klein Spacetime and Noether's Theorem via Lagrangian Multiplier Method. Journal of Modern Physics, 4(6), 2013, pp. 757-760. 
[37] Mohamed S. El Naschie: The hyperbolic Extension of Sigalotti-Hendi-Sharifzadeh's Golden Triangle of Special Theory of Relativity and the Nature of Dark Energy. Journal of Modern Physics, 4(3), 2013, pp. 354-356.

[38] Mohamed S. El Naschie: Topological-Geometrical and Physical Interpretation of the Dark Energy of the Cosmos as a 'Halo' Energy of the Schrodinger Quantum Wave. Journal of Modern Physics, 4(5), 2013, pp. 591-596.

[39] M. S. El Naschie: From Modified Newtonian Gravity to Dark Energy via Quantum Entanglement. Journal of Applied Mathematics and Physics, 2(8), 2014, pp. 803-806.

[40] Ji-Huan He, L. Marek-Crnjac: Mohamed El Naschie's Revision of Albert Einstein's $\mathrm{E}=\mathrm{m} 0 \mathrm{c} 2$ : A Definite Resolution of the Mystery of the Missing Dark Energy of the Cosmos International. Journal of Modern Nonlinear Theory and Application, 2(1), 2013, pp. 55-59.

[41] M. S. El Naschie, L. Marek-Crnjac: Deriving the Exact Percentage of Dark Energy Using a Transfinite Version of Nottale's Scale Relativity. International Journal of Modern Nonlinear Theory and Application, 1(4), 2012, pp. 118-124.

[42] Mohamed S. El Naschie, Atef Helal: Dark Energy Explained via the Hawking-Hartle Quantum Wave and the Topology of Cosmic Crystallography. International Journal of Astronomy and Astrophysics, 3(3), 2013, pp. 318-343.

[43] L. Marek-Crnjac, Mohamed S. El Naschie: Chaotic Fractal Tiling for the Missing Dark Energy and Veneziano Model. Applied Mathematics, 4(11B), 2013, pp. 22-29.

[44] L. Marek-Crnjac, M. S. El Naschie: Quantum Gravity and Dark Energy Using Fractal Planck Scaling. Journal of Modern Physics, 4(11A), 2013, pp. 31-38.

[45] Jean Paul Auffray: E-infinity, the zero set, absolute space and photon spin. Journal of Mondern Physics, 6(5), 2015, pp. 536.

[46] M. S. El Naschie, S. Olsen, J. H. He, S. Nada, L. MarekCrnjac, A. Helal: On the Need for Fractal Logic in High Energy Quantum Physics. International Journal of Modern Nonlinear Theory and Application, 1(3), 2012, pp. 84-92.

[47] Mohamed Salah El Naschie, Leila Marek-Crnjac, Mohamed Atef Helal, Ji-Huan He: A Topological Magueijo-Smolin Varying Speed of Light Theory, the Accelerated Cosmic Expansion and the Dark Energy of Pure Gravity. Applied Mathematics, 5(12), 2014, pp. 1780-1790.

[48] Mohamed S. El Naschie: Compactified dimensions as produced by quantum entanglement, the four dimensionality of Einstein's smooth spacetime and 'tHooft's 4- $\varepsilon$ fractal spacetime. American Journal of Astronomy \& Astrophysics, 2(3), 2014, pp. 34-37.

[49] Mohamed S. El Naschie: Hardy's Entanglement as the Ultimate Explanation for the Observed Cosmic Dark Energy and Accelerated Expansion. International Journal High Energy Physics, 1(2), 2014, pp. 13-17.

[50] Mohamed S. El Naschie: Deriving E = mc2 /22 of Einstein's ordinary quantum relativity energy density from the Lie symmetry group $\mathrm{SO}(10)$ of grand unification of all fundamental forces and without quantum mechanics. American Journal of Mechanics \& Applications, 2(2), 2014, pp. 6-9.
[51] Mohamed S. El Naschie: Cosserat-Cartan Modification of Einstein-Riemann Relativity and Cosmic Dark Energy Density. American Journal of Modern Physics. 3(2), 2014, pp. $82-87$

[52] Mohamed S. El Naschie: Asymptotically safe pure gravity as the source of dark energy of the vacuum. Int. Journal Astrophysics \& Space Science, 2(1), 2014, pp. 12-15.

[53] Mohamed S. El Naschie: Logarithmic Running of 't HooftPolyakov Monopole to Dark Energy. International Journal of High Energy Physics, 1(1), 2014, pp. 1-5.

[54] Mohamed S. El Naschie: Experimentally Based Theoretical Arguments that Unruh's Temperature, Hawking's Vacuum Fluctuation and Rindler's Wedge Are Physically Real. American Journal of Modern Physics, 2(6), 2013, pp. 357-361.

[55] L. Marek-Crnjac: Modification of Einstein's $\mathrm{E}=\mathrm{mc} 2$ to $\mathrm{E}=$ (1/22)mc2. American Journal of Modern Physics, 2(5), 2013, pp. 255-263.

[56] M. S. El Naschie: The quantum gravity Immirzi parameter A general physical and topological interpretation. Gravitation and Cosmology, 19(3), 2013, pp. 151-155.

[57] M. S. El Naschie: Determining the missing dark energy density of the cosmos from a light cone exact relativistic analysis. Journal of Physics, 2(2), 2013, pp. 19-25.

[58] M. S. El Naschie: The quantum entanglement behind the missing dark energy. Journal of Modern Physics and Applications, 2(1), 2013, pp. 88-96.

[59] M. S. El Naschie: Dark energy via quantum field theory in curved spacetime. Journal Modern Physics and Applications, 2, 2014, pp. 1-7.

[60] M. S. El Naschie: Rindler space derivation of dark energy. Journal of Modern Physics Applications. 6, 2014, pp. 1-10.

[61] Wei Tang et al: From nonlocal elasticity to nonlocal spacetime and nano science. Bubbfil Nanotechnology. 1(1), 2014, pp. 3-12.

[62] M. S. El Naschie: To dark energy theory from a Cosserat-like model of spacetime. Problems of Nonlinear Analysis in Engineering Systems. 1(41), Vol. 20, 2014, pp. 79-98.

[63] M. S. El Naschie: Revising Einstein's E = mc2; A theoretical resolution of the mystery of dark energy. Proceedings of the Fourth Arab Int. Conference in Physics and Material Science, Egypt. 1-30 October, 2012, pp. 1.

[64] K. M. Ball: Volume ratios and a reverse isoperimetric inequality. Journal of London Mathematical Society. 44, 1991, pp. 351-359.

[65] G. Pisier: The volume of convex bodies and Banach space geometry. Tracts in Math 94, Cambridge University Press, Cambridge, 1989.

[66] B.S. Kasin: The width of certain finite-dimensional sets and classes of smooth functions. IZV. Akad. Nauk. SSSR. Ser. Mat. 41(2), 1977, pp. 334-351 (in Russian).

[67] O. Guedon: Concentration phenomena in high dimensional geometry. arXiv: 1310.1204V1[math]4 Oct 2013.

[68] Ji-Huan $\mathrm{He}$ (Guest Editor): Fractal Spacetime and Noncommutative Geometry in Quantum and High Energy Physics. 3(1). Special issue on recent developments on dark energy and dark matter, 2013, pp. 1-62. 
[69] Ji-Huan He, M. S. El Naschie: On the monadic nature of quantum gravity as a highly structured golden ring, spaces and spectra. Fractal Spacetime and Noncommutative Geometry in Quantum and High Energy Physics. 2(2), 2012, pp. 94-98.

[70] M. S. El Naschie: Towards a general transfinite set theory for quantum mechanics. Fractal Spacetime and Noncommutative Geometry in Quantum and High Energy Physics. 2(2), 2012, pp. 135-142.

[71] M. S. El Naschie, Ji-Huan He, S. Nada, L. Marek-Crnjac, M. Helal: Golden mean computer for high energy physics. Fractal Spacetime and Noncommutative Geometry in Quantum and High Energy Physics. 2(2), 2012, pp. 80-92.

[72] M. S. El Naschie: The minus one connection of relativity, quantum mechanics and set theory. Fractal Spacetime and Noncommutative Geometry in Quantum and High Energy Physics. 2(2), 2012, pp. 131-134.

[73] M. S. El Naschie: Dark energy and its cosmic density from Einstein's relativity and gauge fields renormalization leading to the possibility of a new 'tHooft quasi particle. The Open Journal of Astronomy. In press.

[74] M. S. El Naschie: A review of E-infinity and the mass spectrum of high energy particle physics. Chaos, Solitons \& Fractals, 19(1), 2004, pp. 209-236.

[75] M. S. El Naschie: The theory of Cantorian spacetime and high energy particle physics (An informal review). Chaos, Solitons \& Fractals, 41, 2009, pp. 2635-2646.

[76] A. Connes: Noncommutative Geometry. Academic Press, San Diego, USA, 1994.

[77] S. G. Krantz and H.R. Parks: Geometric Integration Theory. Birkhauser, Boston, USA, 2008.

[78] M. S. El Naschie: Remarks on super strings, fractal gravity, Nagasawa's diffusion and Cantorian spacetime. Chaos, Solitons \& Fractals, 8(11), 1997, pp. 1873-1886.

[79] M. S. El Naschie: Introduction to nonlinear dynamics, general relativity and the quantum - The uneven flow of fractal time. Chaos, Solitons \& Fractals, 8(5), 1997, pp. vii-x.

[80] M. S. El Naschie: Hyper-dimensional geometry and the nature of physical spacetime. Chaos, Solitons \& Fractals, 10(1), 1999, pp. 155-158.

[81] M. S. El Naschie: E-infinity - High Energy Communications Nos. 1-90. April 2010 to December 2012.

[82] Chen Nanxian: Möbius inversion in physics. World Scientific, Singapore 2010.

[83] Ji-Huan He: A tutorial review on fractal spacetime and fractional calculus. Int. Journal of Theoretical Physics, 53(11), 2014, pp. 3698-3718.

[84] M. S. El Naschie: On twistors in Cantorian E-infinity space. Chaos, Solitons \& Fractals, 12(4), 2001, pp. 741-746.

[85] O. E. Rössler: Endophysics, World Scientific, Singapore (1998).

[86] M. S. El Naschie: On a general theory for quantum gravity. In 'Science of the Interface. Editor H. Diebner, T. Druckrey and P. Weibel. Genista Verlag, Tübingen, Germany, 2001.

[87] Miao Li: A model of holographic dark energy. Physics Letters B, 603(1-2), 2004, pp. 1-5.
[88] M. S. El Naschie: Holographic dimensional reduction. Center manifold theorem and E-infinity. Chaos, Solitons \& Fractals, 29(4), 2006, pp. 816-822.

[89] A. P. Balachandran, S. Kürkcüoglu, S. Vaidya: Lectures on fuzzy and fuzzy Susy physics. World Scientific, Singapore 2007.

[90] J. Bahcall, T. Piran and S. Weinberg (Editors): Dark Matter in the Universe. World Scientific, Singapore (2004).

[91] L. Amendola and S. Tsujikawa: Dark Energy: Theory and Observations. Cambridge University Press, Cambridge 2010.

[92] P. Ruiz-Lapuente, Dark Energy, Observational and Theoretical Approaches. Cambridge University Press, Cambridge, 2010.

[93] M. S. El Naschie: From $E=m c 2$ to $E=m c 2 / 22-$ A short account of the most famous equation in physics and its hidden quantum entangled origin. Journal of Quantum Information Science, 4, 2014, pp. 284-291.

[94] M. S. El Naschie: Casimir-like energy as a double Eigenvalue of quantumly entangled system leading to the missing dark energy density of the cosmos. International Journal of High Energy Physics, 1(5), 2014, pp. 55-63.

[95] S. Perlmutter et al, Supernova cosmology project collaboration "Measurements of omega and lambda from 42 high redshift supernova. Astrophysics Journal, 517, 1999, pp. 565-585.

[96] Mohamed S. El Naschie: On a non-perturbative quantum relativity theory leading to a Casimir-dark energy nanotech reactor proposal. Open Journal of Applied Science, 2015. (Accepted for publication and currently in press).

[97] Vijay Kodiyalam and V. S. Sunder: Topological Quantum Field Theories From Subfactors. Chapma \& Hall/Crc, London, UK, 2001.

[98] M. S. El Naschie: A few hints and some theorem about Witten's M theory and T-duality. Chaos, Solitons \& Fractals, 25(3), 2005, pp. 545-548.

[99] L. Marek-Crnjac, Mohamed S. El Naschie: Ji-Huan He: Chaotic Fractals at the Root of Relativistic Quantum Physics and Cosmology. International Journal of Modern Nonlinear Theory and Application, 2(1A), 2013, pp. 78-88.

[100] S. T. Yau and S. Nadis: The Shape of Inner Space. Basic Book, Persens Group, New York (2010).

[101]J. S. Bell: Speakable and Unspeakable in Quantum Mechanics. Cambridge University Press, Cambridge, 1991.

[102] W. Rindler: Introduction to Special Relativity. Oxford Science Publications, Oxford 1991.

[103] M. S. El Naschie: Hilbert space, Poincaré dodecahedron and golden mean transfiniteness. Chaos, Solitons \& Fractals, 31(4), 2007, pp. 787-793.

[104] Mohamed S. El Naschie: Quantum entanglement as a consequence of a Cantorian micro spacetime geometry. Journal of Quantum Information Science, 1(2), 2011, pp. 50-53.

[105] L. Hardy: Nonlocality of two particles without inequalities for almost all entangled states. Phys. Rev. Lett. 71(11), p. 16651668, 13 September (1993).

[106] D. Mermin: Quantum mysteries refined. American J. Phys. 62(10) (1994). 
[107] P. S. Addison: Fractals and Chaos: An Illustrated Course. Institute of Physics, Bristol, 1997.

[108] D. Ruelle: Chance and Chaos, Princeton University Press, New Jersey, USA, 1991.

[109] L. Graham and Jean-Michel Kantor: Naming Infinity. The Belknap Press of Harvard University Press, Cambridge, Massachusetts-London, 2009.

[110]E. Ott: Chaos in Dynamical Systems, Cambridge University Press, Cambridge, UK, 1993.

[111] T. Kapitaniak (Editor): Chaotic Oscillators (Theory and Applications), World Scientific, Singapore, 1992.

[112] G. Casati, I. Guarneri and U. Smilansky: Quantum Chaos, North Holland, Amsterdam, Netherlands, 1993.

[113] Y. S. Kim and M. E. Noz: Phase Space Picture of Quantum Mechanics, World Scientific, Singapore, 1991.

[114]H. M. Fried: Modern Functional Quantum Field Theory, World Scientific, Singapore, 2014.

[115] M. S. El Naschie: Young double-slit experiment, Heisenberg uncertainity principle and correlation in Cantorian spacetime. In Quantum Mechanics, Diffusion and Chaotic Fractals. Editors M. S. El Naschie, O. E. Rössler and I. Prigogine: Pergamon Press/Elsevier, Oxford ISBN: 0080420273 (1995), pp. 93-100.

[116] M. S. El Naschie: Iterated function system, information and the two-slit experiment of quantum mechanics. In Quantum Mechanics, Diffusion and Chaotic Fractals. Editors M.S. El Naschie, O.E. Rössler and I. Prigogine: Pergamon Press/Elsevier, Oxford ISBN: 0080420273 (1995), pp. 185-189.

[117]M. S. El Naschie: Quantum measurement, information, diffusion and Cantorian geodesic. In Quantum Mechanics, Diffusion and Chaotic Fractals. Editors M.S. El Naschie, O.E. Rössler and I. Prigogine: Pergamon Press/Elsevier, Oxford ISBN: 0080420273 (1995), pp. 191-205.

[118] Mohamed S. El Naschie: Hubble scale dark energy meets nano scale Casimir energy and the rational of their T-duality and mirror symmetry equivalence. World Journal of Nano Science and Engineering, 5, 2015, pp. 57-67.

[119] Mohamed S. El Naschie: An exact mathematical picture of quantum spacetime. Advances in Pure Mathematics, 2015, 5, pp. 560-570.

[120] Mohamed S. El Naschie: From fusion algebra to cold fusion or from pure reason to pragmatism. Open Journal of Philosophy, 2015, 5(6), in press.

[121] Mohamed S. El Naschie: Casimir-dark energy nano reactor design proposal based on fractals. International Journal of Innovation is Science and Mathematics, 3(4), 2015, pp. 187-194.

[122] R. Thom: Structural stability and Morphogenesis. W.A. Benjamin Inc., London, 1975.

[123] M. S. El Naschie: The VAK of vacuum fluctuation, spontaneous self organization and complexity theory interpretation of high energy particle physics and the mass spectrum. Chaos, Solitons \& Fractals, 18(2), 2003, pp. 401-420.

[124]M. S. El Naschie: VAK, vacuum fluctuation and the mass spectrum of high energy physics. Chaos, Solitons \& Fractals, 30(3), 2003, pp. 579-605.
[125] M. S. El Naschie: Stress, Stability and Chaos in Structural Engineering, An Energy Approach. McGraw Hill, London, (1991).

[126] J. M. T. Thompson and G. Hunt: A General Theory of Elastic Stability. J. Wiley, London, 1973.

[127] M. S. El Naschie: A Casimir dark energy nano reactor design - Phase one. Natural Science, 7, 2015, pp. 287-298.

[128] M. S. El Naschie: Cosserat-Cartan and de Sitter-Witten spacetime setting for dark energy. Quantum Matter, 5(1), 2016, pp. 1-4.

[129] M.S. El Naschie: The Cantorian monadic plasma behind the zero point vacuum spacetime energy. American Journal of Nano Research \& Application. 3, 2015, pp. 66-70.

[130] M. S. El Naschie: A nonperturbative quantum relativity theory leading to a Casimir-dark energy nanotech reactor proposal. Open Journal of Applied Science, 5(7), 2015, pp. 313.

[131] Mae-Wan Ho, M. S. El Naschie and MW Giuseppe Vitello: Is spacetime fractal and quantum coherent in the golden mean? Global Journal of Science Frontier Research, 15(1), 2015, pp. $61-80$

[132] M. S. El Naschie: von Neumann geometry and E-infinity quantum spacetime. Chaos, Solitons \& Fractals, 9(12), 1998, pp. 2023-2030.

[133] Clara Moskowitz: Stephen Hawking hasn't solved the black hole paradox just yet. Scientific American, August 27, 2015.

[134] Rachael Feltman: Stephen Hawking believes he's solved a huge mystery about black holes. The Washington Post, August $25,2015$.

[135] Paul Rincon: Hawking: Black holes store information. BBC News, Science \& Environment, 26 August, 2015.

[136] L. Susskind and J. Lindesay: Black Holes, Information and the String Theory Revolution. World Scientific, Singapore, 2005.

[137] L. susskind: The Black Hole Wave. Back Bay Books New York, USA, 2008.

[138] M. S. El Naschie: Fractal black holes and information. Chaos, Solitons \& Fractals, 29(1), 2006, pp. 23-35.

[139] G. 'tHooft: On the quantum structure of a black hole. Nuclear Physics B 256, 1985, pp. 727-745.

[140] Mohamed S. El Naschie: The measure concentration of convex geometry in a quasi Banach spacetime behind the supposedly missing dark energy of the cosmos. American Journal of Astronomy \& Astrophysics, 2014, 2(6), pp. 72-77.

[141] Louis H. Kauffman: Knot Logic. In 'Knots and Applications', Editor H. Kauffman. World Scientific, Singapore, 1995, pp. 1100 .

[142] G. 'tHooft: G. 'tHooft asks a question on Research Gate, Questions and Answers, 29 Sept. 2015. Answered by Mohamed S. El Naschie, 29 Sept. 2015. https://www.researchgate.net/post/In_GR_can_we always_ch oose_the_local_speed_of_light_to_be_everywhere_smaller_th at_the_coordinate_speed_of_light_Can_this_be_used_in_a_th eory. 